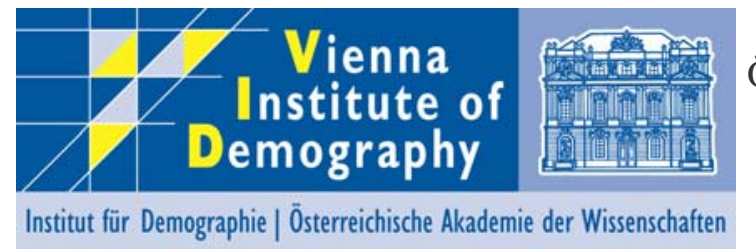

Institut für Demographie Österreichische Akademie der Wissenschaften Prinz Eugen Str. 8-10, 2. OG, 1040 Wien

Tel: +43151581 7702

Email: vid@oeaw.ac.at http://www.oeaw.ac.at/vid

\title{
Neue Projektionen der Bevölkerung in Österreich nach dem Religionsbekenntnis
}

Deutschsprachige Zusammenfassung des Working Paper:

"New Times, Old Beliefs: Projecting the Future Size of Religions in Austria"

Anne Goujon, Vegard Skirbekk, Katrin Fliegenschnee, Pawel Strzelecki

Vienna Institute of Demography, englisches Working Paper, herunterzuladen unter: http://www.oeaw.ac.at/vid/p_vid-workingpapers.shtml

\section{Einleitung}

Die Struktur und Mitgliederzahlen der österreichischen Religionsgemeinschaften haben sich in den letzten Jahrzehnten deutlich verändert. Die römisch-katholische Kirche zählt zwar nach wie vor die meisten Mitglieder, ihre Zahl ist jedoch stark zurückgegangen. Dieselben Tendenzen lassen sich auch in der Evangelischen Kirche erkennen. Die Zahl der Muslime in Österreich sowie der Personen ohne Religionsbekenntnis hat dagegen stark zugenommen. Was bringen die nächsten Jahrzehnte in Hinblick auf die Größe und Altersstruktur der Glaubensgemeinschaften in Österreich? In dieser demographischen Studie wurden Zahlen aus den Volkszählungen von 1981 bis 2001 analysiert. Es wurden die Entwicklung der Einund Austritte, das Fertilitätsverhalten der unterschiedlichen Religionsgemeinschaften sowie die Migration der letzten Jahrzehnte betrachtet. Schließlich wurden alternative Szenarien für die zukünftige Struktur der Glaubensgemeinschaften in Österreich bis zum Jahr 2051 berechnet.

\section{Die Geschichte}

Seit der Gegenreformation um 1600 ist die österreichische Bevölkerung mehrheitlich römisch-katholisch. Juden, Orthodoxe und auch Protestanten gibt es schon lange, sie waren aber immer in der Minderheit. 
Tabelle 1: Bevölkerung auf dem Gebiet der heutigen Republik Österreich nach Religionsgemeinschaft 1900-2001

\begin{tabular}{|c|c|c|c|c|c|c|c|}
\hline Jahr & $\begin{array}{l}\text { Römisch- } \\
\text { katholisch } \\
\text { (\%) }\end{array}$ & $\begin{array}{c}\text { Evangelisch } \\
\text { (\%) }\end{array}$ & $\begin{array}{c}\text { Muslimisch } \\
\text { (\%) }\end{array}$ & $\begin{array}{l}\text { Andere } \\
\text { Religionen } \\
\text { (\%) }\end{array}$ & $\begin{array}{c}\text { Ohne } \\
\text { Religions- } \\
\text { bekenntnis } \\
(\%)\end{array}$ & $\begin{array}{c}\text { Unbekannt } \\
\text { (\%) }\end{array}$ & $\begin{array}{c}\text { Total } \\
\text { (absolut) }\end{array}$ \\
\hline 1900 & 91.6 & 2.7 & 0.0 & 5.4 & 0.2 & 0.2 & $6,003.780$ \\
\hline 1971 & 87.4 & 6.0 & 0.3 & 1. & 4.3 & 0.6 & $7,491.526$ \\
\hline 1981 & 84.3 & 5.6 & 1.0 & 2.0 & 6.0 & 1.0 & $7,555.338$ \\
\hline 1991 & 78.0 & 5.0 & 2.0 & 2.9 & 8.6 & 3.5 & 7,795.786 \\
\hline 2001 & 73.6 & 4.7 & 4.2 & 3.5 & 12.0 & 2.0 & $8,032.926$ \\
\hline
\end{tabular}

Quelle: Statistik Austria, Volkszählung 1900, 1971, 1981, 1991 und 2001

Konnte im Jahr 1900 die römisch-katholische Kirche auf der Fläche des heutigen Österreichs über 91 Prozent der Bevölkerung als Mitglieder zählen, waren es 2001 nur mehr 74 Prozent. Die Gruppe der Muslime ist in den letzten Jahrzehnten stark angewachsen und betrug 2001 bereits 4,2 Prozent. Auch andere Religionsgemeinschaften haben in den letzten Jahren stark zugenommen. Die am stärksten angewachsene Gruppe ist jene ohne religiöses Bekenntnis. Sie betrug 2001 12 Prozent.

\section{Daten}

Für diese Berechnungen wurden Daten aus den Volkszählungen, die in Österreich alle zehn Jahre durchgeführt werden, herangezogen. Bei der Volkszählung handelt es sich um eine Selbstdeklaration, was bedeutet, dass die Befragten selbst das Religionsbekenntnis in den Fragebogen einsetzten. Meist ergibt die Volkszählung eine höhere Zahl von Kirchenmitgliedern als die Kirchen selbst ermitteln, da sich auch Personen, die vielleicht früher katholisch oder evangelisch waren, im Fragebogen als solche angeben, auch wenn sie formell der Kirche nicht mehr angehören. ${ }^{1}$

Es ist zu berücksichtigen, dass die Mitgliederzahlen stark vom jeweiligen Bundesland abhängen. So gibt es in Wien mit Abstand die meisten Personen ohne Religionsbekenntnis. Ebenso zählt man in Wien die meisten muslimischen Einwohner.

\section{Fertilität und Familie}

Die Berechnungen basieren auf der empirischen Tatsache, dass die Geburtenrate mit dem Religionsbekenntnis variiert. Dies ist entscheidend, da sich das über die Zeit auf die Anzahl der Mitglieder niederschlägt. Prinzipiell kann gesagt werden, dass in allen Religionsbekenntnissen sinkende Fertilitätsraten zu beobachten waren. 2001 war mit

1 Es sei bemerkt, dass die Volkszählung 2001 vermutlich die letzte war, in der das Religionsbekenntnis erhoben wurde. In Zukunft wird die Volkszählung durch Registerzählungen auf Basis des Einwohner-Melderegisters ersetzt, wo das Religionsbekenntnis zwar noch auf lokaler Ebene registriert wird (und worin die Kirchen Einsicht nehmen können), aber nicht mehr zentral zusammengeführt wird. 
0,86 Kindern pro Frau die niedrigste Fertilitätsrate bei der Gruppe „ohne religiöses Bekenntnis“ zu finden, die höchste mit 2,34 Kindern bei den muslimischen Frauen.

Ebenfalls eine Auswirkung auf die Mitgliederzahlen haben gemischtkonfessionelle Ehen. Bei mehr als 82 Prozent aller evangelischen Frauen wird die Frage nach der Konfession ihrer Kinder wegen eines anderskonfessionellen oder konfessionslosen Partners zumindest zum Diskussionspunkt. Im Vergleich dazu heiraten rund 77 Prozent der römisch-katholischen Frauen einen Mann der gleichen Konfession und 84 Prozent der weiblichen muslimischen Bevölkerung ebenso einen muslimischen Partner. Die Frage, inwieweit gemischtkonfessionelle Paare eine Bedeutung für die Mitgliederzahlen haben, wie etwa in der Evangelischen Kirche, hängt stark von der relativen Größe der jeweils betrachteten Glaubensgemeinschaft ab.

\section{Kirchenaustritte}

In westlichen Ländern verlässt eine große Anzahl von Mitgliedern die christlichen Kirchen und kann keiner Glaubensgemeinschaft mehr zugeordnet werden. Dieses Phänomen lässt sich nicht nur in Österreich, sondern auch in vielen anderen europäischen Ländern beobachten. Im jährlichen Saldo der Ein- und Austritte schrumpft die römisch-katholische ebenso wie auch die Evangelische Kirche in Österreich. Die Zahlen für die römisch-katholische Kirche belaufen sich jährlich auf etwa 30.000 Mitglieder.

\section{$\underline{\text { 6. Migration }}$}

Ein weiteres Schlüsselelement ist die Nettomigration nach Österreich. Daten darüber, welches Religionsbekenntnis MigrantInnen haben, waren für dieser Studie leider nicht zugänglich. Daher wurden Schätzungen vorgenommen, die sich an den Herkunftsländern der MigrantInnen und den dort vorherrschenden Religionsgemeinschaften orientierten. Laut diesen Berechnungen waren 200118 Prozent der Personen römisch-katholisch, acht Prozent protestantisch, 38 Prozent muslimisch, 28 Prozent hatten ein anderes Religionsbekenntnis, und neun Prozent waren ohne Bekenntnis.

\section{Szenarien}

Aus demographischer Sicht wird die zukünftige Entwicklung der Struktur der österreichischen Glaubenslandschaft durch die Veränderungen der weiter oben beschriebenen Faktoren bestimmt werden. Zusätzlich zur vorgegebenen bestehenden Struktur kommt es auf die Bilanz von Ein- und Austritten, den Gewinn oder Verlust durch internationale Migration sowie auf die religionsspezifische Fertilität an.

Auf diesen Annahmen beruhen die Berechnungen für die Szenarien. Um das Feld möglicher Entwicklungen abzustecken, werden daher verschiedene Annahmen getroffen. Die Kombination alternativer Bündel von Annahmen nennt man Szenarien. Solche Szenarien bestimmen nicht, welche Entwicklung wahrscheinlicher ist als eine andere, sie zeigen lediglich, was die langfristigen Folgen möglicher Entwicklungen sind. Sie liefern somit eine Basis für die Beurteilung unterschiedlicher möglicher Entwicklungen für die nächsten Jahre. Es wurden 36 unterschiedliche Szenarien errechnet und von diesen sechs exemplarisch herausgegriffen und dargestellt. 


\section{Szenario}

Folgende Annahmen wurden getroffen:

- Die Fertilitätsraten wurden stabil gehalten, auf dem Niveau, wie sie 2001 beobachtet wurden.

- Es wurde auf die mittlere Variante der Migrationsprognosen der Statistik Austria zurückgegriffen.

- Die Austrittsmuster, die 2001 beobachtet werden konnten, sind in diesem Szenario unverändert für die gesamte berechnete Periode angenommen worden.

Die Ergebnisse sind:

Tabelle 2: Bevölkerung nach Alter und Religion 2001 und 2051 in Prozent

\begin{tabular}{|c|c|c|c|c|c|c|c|c|c|}
\hline \multirow[b]{2}{*}{ Jahr } & \multirow[b]{2}{*}{ Fertilität } & \multirow[b]{2}{*}{ Migration } & \multirow[b]{2}{*}{ Austritte } & \multirow{2}{*}{$\begin{array}{l}\text { Alters- } \\
\text { gruppe }\end{array}$} & \multicolumn{5}{|c|}{ Religion } \\
\hline & & & & & Katholisch & Protestantisch & Muslimisch & Andere & Ohne \\
\hline & & \multirow{3}{*}{\multicolumn{2}{|c|}{2001}} & $0-14$ & 78.2 & 4.2 & 7.4 & 5.4 & 4.7 \\
\hline & & & & 15-64 & 71.7 & 4.6 & 4.3 & 6.0 & 13.4 \\
\hline & & & & $65+$ & 77.1 & 5.7 & 0.4 & 3.0 & 13.8 \\
\hline \multirow{3}{*}{2051} & Stabil & Medium & Konstant & $0-14$ & 40.7 & 4.0 & 33.6 & 12.5 & 9.3 \\
\hline & Stabil & Medium & Konstant & $15-64$ & 42.3 & 4.0 & 19.5 & 11.7 & 22.6 \\
\hline & Stabil & Medium & Konstant & $65+$ & 55.6 & 3.3 & 6.9 & 7.0 & 27.3 \\
\hline
\end{tabular}

\section{Szenario}

Folgende Annahmen wurden getroffen:

- Die Fertilitätsraten wurden stabil gehalten, auf dem Niveau, wie sie 2001 beobachtet wurden.

- Es wurde angenommen, dass nur Moslems nach Österreich immigrieren, um zu sehen, welche Auswirkungen dies auf die Bevölkerungsstruktur hätte.

- Es wurde die Verdoppelung der Austrittsrate, bezogen auf den jeweiligen Mitgliederstand, angenommen.

Die Berechnungen ergeben:

Tabelle 3: Bevölkerung nach Alter und Religion 2001 und 2051 in Prozent

\begin{tabular}{|c|c|c|c|c|c|c|c|c|c|}
\hline \multirow[b]{2}{*}{ Jahr } & \multirow[b]{2}{*}{ Fertilität } & \multirow[b]{2}{*}{ Migration } & \multirow[b]{2}{*}{ Austritte } & \multirow{2}{*}{$\begin{array}{l}\text { Alters- } \\
\text { gruppe }\end{array}$} & \multicolumn{5}{|c|}{ Religion } \\
\hline & & & & & Katholisch & Protestantisch & Muslimisch & Andere & Ohne \\
\hline \multirow{3}{*}{\multicolumn{4}{|c|}{2001}} & $0-14$ & 78.2 & 4.2 & 7.4 & 5.4 & 4.7 \\
\hline & & & & $15-64$ & 71.7 & 4.6 & 4.3 & 6.0 & 13.4 \\
\hline & & & & $65^{+}$ & 77.1 & 5.7 & 0.4 & 3.0 & 13.8 \\
\hline \multirow{3}{*}{2051} & Stabil & Muslime & Hoch & $0-14$ & 29.5 & 2.6 & 51.3 & 6.0 & 10.6 \\
\hline & Stabil & Muslime & Hoch & $15-64$ & 29.7 & 3.5 & 30.6 & 7.0 & 29.2 \\
\hline & Stabil & Muslime & Hoch & $65+$ & 49.9 & 4.4 & 6.0 & 7.3 & 32.4 \\
\hline
\end{tabular}




\section{Szenario}

Folgende Annahmen wurden getroffen:

- Die Fertilitätsraten wurden so angenommen, dass sich die Geburtenraten derer, die wenige, und jener, die viele Kinder bekommen, bis 2031 angleichen und fortan für alle auf dem gleichen Niveau bleiben.

- Es wurde auf die mittlere Variante der Migrationsprognosen der Statistik Austria zurückgegriffen.

- Es wurde davon ausgegangen, dass sich die Austrittsraten in Zukunft halbieren werden.

Die Berechnungen ergeben:

Tabelle 4: Bevölkerung nach Alter und Religion 2001 und 2051 in Prozent

\begin{tabular}{|c|c|c|c|c|c|c|c|c|c|}
\hline \multirow[b]{2}{*}{ Jahr } & \multirow[b]{2}{*}{ Fertilität } & \multirow[b]{2}{*}{ Migration } & \multirow[b]{2}{*}{ Austritte } & \multirow{2}{*}{$\begin{array}{l}\text { Alters- } \\
\text { gruppe }\end{array}$} & \multicolumn{5}{|c|}{ Religion } \\
\hline & & & & & Katholisch & Protestantisch & Muslimisch & Andere & Ohne \\
\hline \multirow{3}{*}{\multicolumn{4}{|c|}{2001}} & $0-14$ & 78.2 & 4.2 & 7.4 & 5.4 & 4.7 \\
\hline & & & & $15-64$ & 71.7 & 4.6 & 4.3 & 6.0 & 13.4 \\
\hline & & & & $65+$ & 77.1 & 5.7 & 0.4 & 3.0 & 13.8 \\
\hline \multirow{3}{*}{2051} & Angleichung & Medium & Niedrig & $0-14$ & 54.1 & 5.3 & 18.9 & 12.5 & 9.2 \\
\hline & Angleichung & Medium & Niedrig & $15-64$ & 56.2 & 5.0 & 16.9 & 11.6 & 10.3 \\
\hline & Angleichung & Medium & Niedrig & $65+$ & 62.4 & 4.0 & 6.9 & 7.0 & 19.7 \\
\hline
\end{tabular}

\section{Szenario}

Folgende Annahmen wurden getroffen:

- Die Fertilitätsraten wurden so angenommen, dass sich die Geburtenraten derer, die wenige, und jener, die viele Kinder bekommen, bis 2031 angleichen und fortan für alle auf dem gleichen Niveau bleiben.

- Es wurde auf die Variante der Prognosen der Statistik Austria zurückgegriffen, in der von hoher Migration ausgegangen wird.

- Es wurde davon ausgegangen, dass auch muslimische Personen austreten werden. Dabei ging man von den gleichen Raten wie in der römischkatholischen Kirche aus.

Die Berechnungen ergeben:

Tabelle 5: Bevölkerung nach Alter und Religion 2001 und 2051 in Prozent

\begin{tabular}{|c|c|c|c|c|c|c|c|c|c|}
\hline \multirow[b]{2}{*}{ Jahr } & \multirow[b]{2}{*}{ Fertilität } & \multirow[b]{2}{*}{ Migration } & \multirow[b]{2}{*}{ Austritte } & \multirow{2}{*}{$\begin{array}{l}\text { Alters- } \\
\text { gruppe }\end{array}$} & \multicolumn{5}{|c|}{ Religion } \\
\hline & & & & & Katholisch & Protestantisch & Muslimisch & Andere & Ohne \\
\hline \multirow{3}{*}{\multicolumn{4}{|c|}{2001}} & $0-14$ & 78.2 & 4.2 & 7.4 & 5.4 & 4.7 \\
\hline & & & & $15-64$ & 71.7 & 4.6 & 4.3 & 6.0 & 13.4 \\
\hline & & & & $65+$ & 77.1 & 5.7 & 0.4 & 3.0 & 13.8 \\
\hline \multirow[b]{2}{*}{2051} & Angleichung & Hoch & Muslime & $0-14$ & 50.1 & 3.4 & 33.0 & 6.3 & 7.2 \\
\hline & Angleichung & Hoch & Muslime & $15-64$ & $\overline{53.3}$ & 3.6 & 27.4 & 6.9 & 8.9 \\
\hline
\end{tabular}




\begin{tabular}{|c|c|c|c|c|c|c|c|c|c|}
\hline \multirow[b]{2}{*}{ Jahr } & \multirow[b]{2}{*}{ Fertilität } & \multirow[b]{2}{*}{ Migration } & \multirow[b]{2}{*}{ Austritte } & \multirow{2}{*}{$\begin{array}{l}\text { Alters- } \\
\text { gruppe }\end{array}$} & \multicolumn{5}{|c|}{ Religion } \\
\hline & & & & & Katholisch & Protestantisch & Muslimisch & Andere & Ohne \\
\hline \multirow{3}{*}{\multicolumn{4}{|c|}{2001}} & $0-14$ & 78.2 & 4.2 & 7.4 & 5.4 & 4.7 \\
\hline & & & & 15-64 & 71.7 & 4.6 & 4.3 & 6.0 & 13.4 \\
\hline & & & & $65+$ & 77.1 & 5.7 & 0.4 & 3.0 & 13.8 \\
\hline & Angleichung & Hoch & Muslime & $65^{+}$ & 62.7 & 4.1 & 6.0 & 7.3 & 19.9 \\
\hline
\end{tabular}

\section{Szenario}

Folgende Annahmen wurden getroffen:

- Die Fertilitätsraten wurden so angenommen, dass sich die Geburtenraten annähern, aber ab 2031 zur Hälfte dieser Annäherung konstant bleiben.

- Es wurde angenommen, dass nur Moslems nach Österreich immigrieren, um zu sehen, welche Auswirkungen dies auf die Bevölkerungsstruktur hätte.

- Es wurde die Verdoppelung der Austrittsrate, bezogen auf den jeweiligen Mitgliederstand, angenommen.

Die Berechnungen ergeben:

Tabelle 6: Bevölkerung nach Alter und Religion 2001 und 2051 in Prozent

\begin{tabular}{|c|c|c|c|c|c|c|c|c|c|}
\hline \multirow[b]{2}{*}{ Jahr } & \multirow[b]{2}{*}{ Fertilität } & \multirow[b]{2}{*}{ Migration } & \multirow[b]{2}{*}{ Austritte } & \multirow{2}{*}{$\begin{array}{l}\text { Alters- } \\
\text { gruppe }\end{array}$} & \multicolumn{5}{|c|}{ Religion } \\
\hline & & & & & Katholisch & Protestantisch & Muslimisch & Andere & Ohne \\
\hline \multirow{3}{*}{\multicolumn{4}{|c|}{2001}} & $0-14$ & 78.2 & 4.2 & 7.4 & 5.4 & 4.7 \\
\hline & & & & $15-64$ & 71.7 & 4.6 & 4.3 & 6.0 & 13.4 \\
\hline & & & & $65+$ & 77.1 & 5.7 & 0.4 & 3.0 & 13.8 \\
\hline \multirow[t]{3}{*}{2051} & $\begin{array}{l}\text { Halbe } \\
\text { Angleichung }\end{array}$ & Muslime & Hoch & $0-14$ & 32.7 & 3.0 & 42.5 & 6.2 & 15.5 \\
\hline & $\begin{array}{l}\text { Halbe } \\
\text { Angleichung }\end{array}$ & Muslime & Hoch & $15-64$ & 30.1 & 3.6 & 29.0 & 6.9 & 30.3 \\
\hline & $\begin{array}{l}\text { Halbe } \\
\text { Angleichung }\end{array}$ & Muslime & Hoch & $65^{+}$ & 49.9 & 4.4 & 6.0 & 7.3 & 32.4 \\
\hline
\end{tabular}

\section{Szenario}

Folgende Annahmen wurden getroffen:

- Die Fertilitätsraten wurden so angenommen, dass sich die Geburtenraten annähern, aber ab 2031 zur Hälfte dieser Annäherung konstant bleiben.

- Es wurde auf die Variante der Prognosen der Statistik Austria zurückgegriffen, in der von hoher Migration ausgegangen wird.

- Es wurde davon ausgegangen, dass auch muslimische Personen austreten werden. Dabei ging man von den gleichen Raten wie in der römischkatholischen Kirche aus. 
Die Berechnungen ergeben:

Tabelle 7: Bevölkerung nach Alter und Religion 2001 und 2051 in Prozent

\begin{tabular}{|c|c|c|c|c|c|c|c|c|c|}
\hline \multirow[b]{2}{*}{ Jahr } & \multirow[b]{2}{*}{ Fertilität } & \multirow[b]{2}{*}{ Migration } & \multirow[b]{2}{*}{ Austritte } & \multirow{2}{*}{$\begin{array}{l}\text { Alters- } \\
\text { gruppe }\end{array}$} & \multicolumn{5}{|c|}{ Religion } \\
\hline & & & & & Katholisch & Protestantisch & Muslimisch & Andere & Ohne \\
\hline \multirow{3}{*}{\multicolumn{4}{|c|}{2001}} & $0-14$ & 78.2 & 4.2 & 7.4 & 5.4 & 4.7 \\
\hline & & & & $15-64$ & 71.7 & 4.6 & 4.3 & 6.0 & 13.4 \\
\hline & & & & $65+$ & 77.1 & 5.7 & 0.4 & 3.0 & 13.8 \\
\hline \multirow[t]{3}{*}{2051} & $\begin{array}{l}\text { Halbe } \\
\text { Angleichung }\end{array}$ & Hoch & Muslime & $0-14$ & 46.7 & 5.0 & 27.9 & 13.7 & 6.6 \\
\hline & $\begin{array}{l}\text { Halbe } \\
\text { Angleichung }\end{array}$ & Hoch & Muslime & $15-64$ & 52.2 & 5.2 & 20.1 & 13.0 & 9.6 \\
\hline & $\begin{array}{l}\text { Halbe } \\
\text { Angleichung }\end{array}$ & Hoch & Muslime & $65+$ & 62.0 & 4.0 & 7.2 & 7.2 & 19.7 \\
\hline
\end{tabular}

\section{Konklusion}

Die Struktur der Glaubensgemeinschaften in Österreich hat sich in den letzten Jahren verändert. Viele Menschen sind aus den christlichen Kirchen ausgetreten, und durch die Migration vergangener Jahre sind die Religionsgemeinschaften vielfältiger geworden. Stark hat die Gruppe der Muslime und der Personen ohne Bekenntnis zugenommen.

Diese Tendenzen sind auch für die kommenden Jahre durchaus realistische Annahmen. Wie die berechneten Szenarien zeigen, ist jedoch ein großer Spielraum gegeben. Je nachdem, wie sich Ein- und Austritte sowie die Fertilität und die Migration verschiedener Gruppen entwickeln, sind schwache oder starke Veränderungen in der Mitgliederzahl der einzelnen Religionsgemeinschaften vorstellbar.

Die Szenarien ergaben unterschiedliche Auswirkungen auf die Bevölkerungszusammensetzung. In allen Berechnungen ist ein weiterer Rückgang der Mitglieder der römisch-katholischen Kirche zu sehen. Die Schere bewegt sich zwischen 60 und 35 Prozent. Dennoch bleiben die KatholikInnen in allen Szenarien die größte Gruppe. Für die Protestanten ist die Zukunft ziemlich unsicher, da die Migration die Mitgliederzahlen stark beeinflussen kann. Sicherlich wird die Gruppe der Moslems am stärksten anwachsen. Dennoch lässt sich erkennen, dass sie selbst in unrealistischen, extremen Szenarien nur 26 Prozent erreichen werden. Realistischer sind aber 14 bis 20 Prozent. In jedem Fall erweitert Migration die Vielfältigkeit der Religionen in Österreich. Die Gruppe der ,anderen Religionen“ wird auch in Zukunft stark zunehmen (mit meist zehn bis zwölf Prozent errechnet). Die zweitgrößte Gruppe Mitte dieses Jahrhunderts wird die „ohne Religionsbekenntnis“ sein. Hier ist eine große Bandbreite zu erkennen, da dies stark von weiteren Austritten abhängt. So könnte diese Gruppe zwölf, aber auch bis zu 30 Prozent der Bevölkerung ausmachen. Für alle Szenarien gilt, dass besonders für Personen zwischen 15 und 64 Jahren sowie auch für Kinder unter 14 Jahren eine starke Durchmischung von Religionsbekenntnissen zu erwarten sein wird. 
Wie es sich im Detail entwickeln wird, bleibt abzuwarten. Dennoch sind solche Blicke in die Zukunft hilfreich, um besser auf Veränderungen eingehen zu können. In Zukunft plant man weitere Berechungen durchzuführen, wie etwa in der Schweiz und in Kanada, um zu sehen, wie sich dort die Zusammensetzung von Religionsgemeinschaften verändern könnte.

Kontakt: Mag. Katrin Fliegenschnee

Tel: +431515 817710

Email: katrin.fliegenschnee@oeaw.ac.at

Prof. Wolfgang Lutz

Tel: +432236 807294

Email: lutz@iiasa.ac.at 